\title{
Intracystic Papillary Carcinoma in a Male Breast Cyst: A case report
}

\author{
${ }^{1}$ Dr. Sathish Babu N, ${ }^{2}$ Dr. chandrashekar N \\ ${ }^{I}$ Assistant professor, Dept. of General surgery/SSMC/SAHE, Tumkur/India \\ ${ }^{2}$ Associate professor, Dept. of General surgery/SSMC/SAHE, Tumkur/India
}

\begin{abstract}
Intracystic papillary breast carcinoma is a rare form of non-invasive carcinoma with an excellent prognosis. It accounts for less than $0.5 \%$ of breast cancers. We report the case of a 60 year old man presenting with a painless cystic lump in the left breast. Ultrasonography showed a cystic lesion and aspiration revealed benign fibrocystic lesion. Excisional biopsy was necessary to confirm the diagnosis and also indicated that local treatment was adequate.
\end{abstract}

Keywords: Intracystic, Papillary carcinoma, Male breast cancer

\section{Introduction}

Male breast cancer is a rare disease and the incidence is $1 \%$ of all breast cancer. Intracystic papillary breast carcinoma is an extremely rare condition in men and represents only $5-7.5 \%$ of all male breast carcinomas. Diagnosis is often delayed because of the benign appearance on physical examination and radiological imaging. Such carcinomas have a better prognosis than other types of breast malignancy. Fine needle aspiration is often inaccurate and excision biopsy may be necessary for the diagnosis. We report a case of 60-year-old man presenting with a cystic lesion of the left breast after an excisional biopsy confirmed the diagnosis and also indicated that local treatment was adequate.

\section{Case Report}

A 60-year-old male presented with a painless mass in the left breast of 2-years duration. On examination, he had a well-defined $9 \mathrm{~cm} \times 10 \mathrm{~cm}$, cystic, non-tender, non-adhering lump occupying whole of the central compartment of the left breast (picture 1). The right breast and both axillae were normal. Ultrasound showed a cyst. Aspiration of the lump revealed dark brown fluid. Microscopic examination revealed foamy macrophages in addition to numerous inflammatory cells and RBC's against fluid background. Ductal epithelial cells are not seen, the patient subsequently underwent a lumpectomy (picture.2)

Macroscopic examination of the specimen showed a cyst measuring $10 \times 6 \mathrm{cms}$ of diameter with an intracystic fleshy papillary lesion in its wall. Multiple sections were taken for microscopic examination. The cyst wall was lined by a single layer of epithelial cells without atypia. The papillary lesion was a non-invasive papillary carcinoma. The carcinoma cells were round to polyhedral with mild atypia and rare mitoses. There was no evidence of stromal invasion. The tumour was diagnosed as intracystic papillary carcinoma of low grade excised margins were free of tumour. No other treatment was performed. He was doing well after 6 months follow-up. Microscopic examination shows a well-defined cystic lesion with a fleshy papillary lesion in its wall. Histology shows a cyst containing a papillary and glandular lesion on its wall with extensive microcalcification. The tumour consists of round to polyhedral epithelial cells. Nuclear atypia is mild and mitoses are rare and no evidence of stromal invasion. (picture.3)

\section{Discussion}

Intracystic breast carcinoma is rare in females and exceedingly rare in males with a handful of case reports in the literature It accounts for 5-7.5\% of all breast cancers in males It is a localised, non-invasive breast cancer with papillary proliferation arising within or on the wall of a large cyst

It commonly presents as a benign-appearing, well-localised lump due to its underlying cystic nature. In a case report and subsequent review of Japanese cases in men, Tochika reported the mean age of intracystic carcinoma in males as 68.2 years. Most of the patients presented with a palpable lump. In addition to abnormalities felt on palpation, a few patients presented with mild pain, bloody nipple discharge and pruritus.

Radiological studies are helpful. Ultrasonography of these lesions typically reveals a hypo-echoic area (representing the cyst) with soft tissue echoes projecting from wall of the cyst (intracystic tumour). Biopsy should be aggressively performed under a tentative diagnosis of intracystic breast carcinoma when ultrasound reveals cyst with mural nodules and aspiration fluid is bloody. Fine needle aspiration is rarely diagnostic and an excisional biopsy is usually required to establish the diagnosis. Intracystic papillary carcinoma tends to be welldefined on mammography; an irregular margin suggests the presence of invasion. 


\section{Conclusions}

Intracystic papillary breast carcinoma is extremely rare in males. Clinically it presents as a benignlooking cystic lesion. Mammographic appearance is typically of benign, well-circumscribed, mass lesion. The diagnosis of an intracystic malignancy should be suspected when ultrasonography shows intracystic mural nodule, the mass persists or recurs after fine needle aspiration or cellular atypia is identified in the aspirate specimen. Wide, local excision without axillary dissection is currently the treatment of choice and the prognosis is favorable.

\section{References}

[1]. Gupta D, Torosian MH. Intracystic breast carcinoma in male: unusual case presentation and literature review. Oncol Reports. 2002; 9:405-7.

[2]. Andres B, Aguilar J, Torroba A, Martinez-Galvez M, Aguayo J. Intracystic papillary carcinoma in the male breast. Breast J. 2003; 9:249-50.

[3]. Tochika N, Takano A, Yoshimoto T, Tanaka J, Sugimoto T, Kobayashi M, et al. Intracystic carcinoma of the male breast: report of a case. Surg Today. 2001; 31:806-9.

[4]. Jones BM, Bradbeer JW. The presentation and progress of macroscopic breast cyst. Br J Surg. 1980;67:669-71

[5]. Estabrook A, Asch T, Gump F, Kister SJ, Geller P. Mamographic features of intracystic papillary lesion. Surg Gynecol Obstet. 1990; 170:113-6.

[6]. Tsuda H, Uei Y, Fukutomi T, Hirohashi S. Different incidence of loss of heterozygosity on chromosome 16q between intraductal papilloma and intracystic papillary carcinoma of the breast. Jpn J Cancer Res. 1994;85:992-6.

[7]. Harris KP, Faliakou EC, Exon DJ, Nasiri N, Sacks NP, Gui GP. Treatment and outcome of intracystic papillary carcinoma of the breast. Br J Surg. 1999; 86:1274

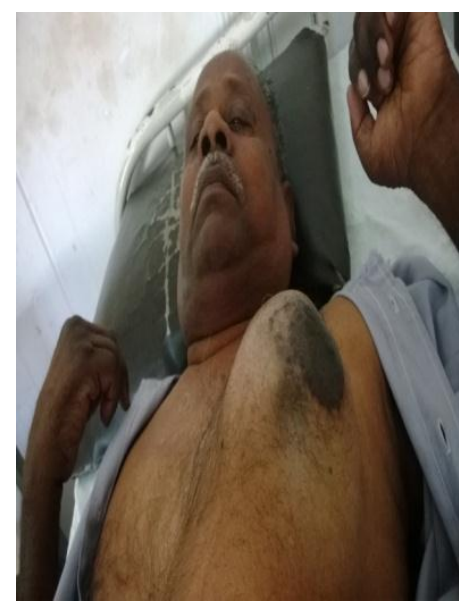

( Picture.1) Picture Showing Swelling Of Left Breast.

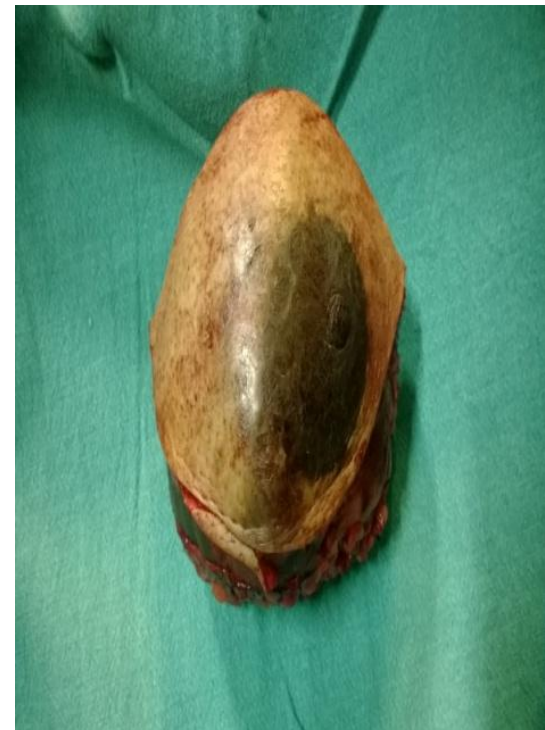

(Picture.2) Picture Showing Excised Specimen of Left Breast 


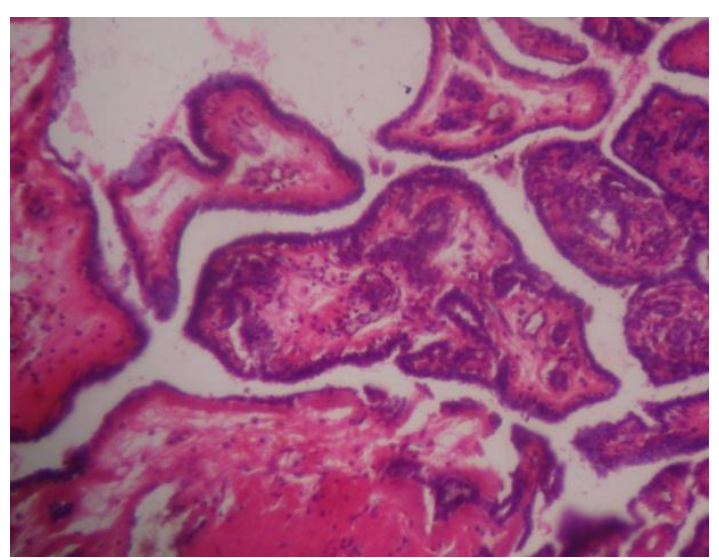

(Picture.3) Histopathology Showing Tumor Cells 